\title{
扬子崆岭高级变质地体古元古代基性岩脉的发现 及其意义
}

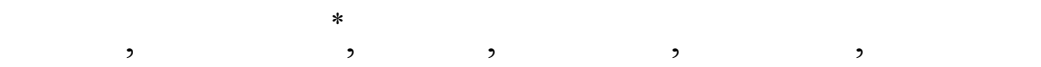

(1) 地质过程与矿产资源国家重点实验室, 中国地质大学地球科学学院, 武汉 430074;

(2) 大陆动力学国家重点实验室, 西北大学地质学系, 西安 710069

* 联系人, E-mail: cugybwu@yahoo.com

2008-08-05 收稿, 2008-10-20 接受

国家自然科学基金(批准号: 40772042, 90714010 和 40521001)、教育部项目(编号: IRT0441, B07039 和 NCET-06-0659)和西北大学大陆动力 学国家重点实验室开放基金资助项目

Peng M, Wu Y B, Wang J, et al. Paleoproterozoic mafic dyke from Kongling terrain in the Yangtze Craton and its implication.

Chinese Science Bulletin, 2009, 54, doi: 10.1007/s11434-008-0558-0

摘要 基性岩脉是区域伸展作用的标志, 具有明确的构造指示意义. 扬子板块崆岭高级变质 地体内部发育有大量的基性岩脉, 这些基性岩脉对于认识扬子板块早期的构造演化具有重要 意义; 但是, 目前还没有关于其形成年龄的报道. 对侵入崆岭变质地体内部的一个辉绿岩脉 样品进行了 LA-(MC)ICP-MS 锆石 U-Pb 定年和 Hf 同位素分析, 得到近似谐和或谐和年龄, 它 们的 ${ }^{207} \mathrm{~Pb} /{ }^{206} \mathrm{~Pb}$ 年龄加权平均结果为 $(1852 \pm 11) \mathrm{Ma}(\mathrm{MSWD}=0.37$ ), 代表了该基性岩脉的形 成年龄; 该基性岩脉中锆石的 $\varepsilon_{\mathrm{Hf}}(t)$ 为 $-6.3 \sim 0.5$, 加权平均值为 $(-3.06 \pm 0.88)(\mathrm{MSWD}=1.01)$, 显示其岩浆源区可能为受到交代作用影响的岩石圈地幔. 这一发现表明, 在约 $1850 \mathrm{Ma}$ 扬子 板块处于碰撞后板内伸展构造环境中, 同时该时期扬子板块已经具备足够的刚性, 以致能产 生大规模的脆性破裂以及可能引发地幔对流作用, 因此我们推测在约 $1.85 \mathrm{Ga}$ 扬子板块已经 具有克拉通的性质.

关键词

崆岭高级变质体

古元古代

基性岩脉

锆石 $\mathrm{U}-\mathrm{Pb}$ 定年

伸展构造

克拉通化
基性岩脉(墙)主要由源自地幔的玄武质岩浆沿 张性裂隙贯入而形成 ${ }^{[1]}$ ，通常被作为区域伸展作用的

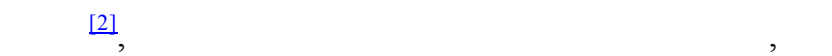
蕴含着有关古大陆聚合和分离时代及过程的重要信 息, 因此具有明确的构造及地球动力学意义 ${ }^{[3]}$. 统计 全球基性岩脉的资料显示, 地球演化过程中全球范 围内存在多期基性岩浆活动, 这些基性岩浆活动可 能与全球周期性超大陆的聚合和裂解以及地幔物质对 流有关 ${ }^{[4.5]}$. 其中, 中元古代-古元古代的基性岩脉(墙) 为地球演化过程中一期重要的基性岩浆事件, 它们广 泛出露于各古老克拉通内, 是中元古代-古元古代大 陆岩石圈演化中重要而独具特色的构造单元, 对于研 究中元古代-古元古代地球演化具有重要意义 ${ }^{[5 \sim 7]}$.
华北板块和扬子板块是中国最大的两个前寒武 板块, 两者于三叠纪沿秦岭-大别造山带碰撞在一起 [8 10]. 华北板块广泛出露太古宙的岩石, 同时有大量 与Columbia超大陆聚合和裂解有关的大规模的古元 古代构造热事件的记录 ${ }^{[11 ~ 18]}$. 但是, 扬子仅少量地方 出露太古宙基底, 有关其与Columbia超大陆聚合和 裂解相关的研究结果报道较少. 最新研究表明, 扬子 板块不同地方存在 1.80 2.0 Ga的构造热事件的年代 学记录 ${ }^{[19-24]}$, 这些记录表明扬子板块存在时代约为 $2.0 \mathrm{Ga}$ 的碰撞造山事件, 并且可能与Columbia超大陆 的聚合有关 ${ }^{[23,24]}$. 但是, 前人的研究对象主要为扬子 板块 $2.0 \mathrm{Ga}$ 碰撞造山事件中高级变质作用的时代, 关 于该时期的岩浆作用及扬子板块碰撞后的演化的研 
究较为薄弱，从而限制了对扬子板块早期演化历史 的认识.

崆岭地区太古宙变质基底中有大量的基性岩脉, 虽然规模较小，但是准确测定它们的侵入年龄对于 认识扬子板块早期构造演化历史具有重要的意义. 然而，目前还没有这些基性岩脉形成年龄的相关报 道. 本文首次对崆岭变质地体中一个辉绿岩脉进行 了 LA-(MC)ICP-MS 锆石 U-Pb 定年和 Hf 同位素分析, 结果为认识扬子板块古元古代构造演化历史和扬子 板块的克拉通化时限提供了重要的制约.

\section{1 研究区概况与样品描述}

崆岭高级变质体位于扬子板块北缘宜昌黄陵地 区䄰归-兴山一带, 呈一穹鄀状产出, 面积约 360 $\mathrm{km}^{2[25]}$. 其北部有时代约为 $1.85 \mathrm{Ga}$ 的圈椅埫钾长花 岗岩侵入其中 ${ }^{[26]}$, 南部为大规模的新元古代黄陵花 岗岩 ${ }^{[27]}$ (图 1). 崆岭高级变质体主体主要由 3 套岩石 组合构成: (1) 闪长质-英云闪长质-奥长花岗质-花岗 闪长质(DTTG)片麻岩和花岗质片麻岩 $(51 \%)$; (2) 变 沉积岩 (44\%); (3) 少量斜长角闪岩和基性麻粒岩 (5\%), 它们呈透镜状、布丁状或似层状产出于长英质
片麻岩中. 其中, DTTG质片麻岩和花岗质片麻岩主 要以奥长花岗质片麻岩为主; 变沉积岩主要为含或 不含石墨的条带状黑云变粒岩和片麻岩, 显示出典 型的孔兹岩建造特征 ${ }^{[25]}$.

在上述的太古宙变质基底中，有大量的基性岩 脉产出, 总体为北北西走向, 倾角接近于 $90^{\circ}$. 这些 岩脉宽 $0.4 \sim 3 \mathrm{~m}$, 长 1 5 km, 岩性主要为辉绿岩或辉 长岩, 无明显的变质变形特征. 本文研究的辉绿岩脉 06HL33 采集于殷家坪到坦荡河之间的公路旁 $\left(31^{\circ} 11^{\prime} 03.9^{\prime \prime} \mathrm{N}, 111^{\circ} 11^{\prime} 01.2^{\prime \prime} \mathrm{E}\right)$, 岩脉宽约 $0.5 \sim 1 \mathrm{~m}$, 围岩为英云闪长岩, 该岩脉具典型的辉绿结构, 粒度 较细, 主要造岩矿物为辉石和斜长石, 另有少量角闪 石、黑云母和钛铁矿.

\section{2 分析方法}

样品 06HL33 中的锆石采用标准重矿物分离技术 (包括磁选和重液分选等)分选, 然后在双目显微镜下 选择透明、无裂隙且具有代表性的锆石颗粒制成环氧 树脂样品靶, 磨至锆石颗粒中心部位后抛光, 样品抛 光后进行 CL 显微结构观察，在此基础上选择合适的 锆石颗粒和/或区域进行 U-Pb 年龄测定. 锆石的 $\mathrm{CL}$

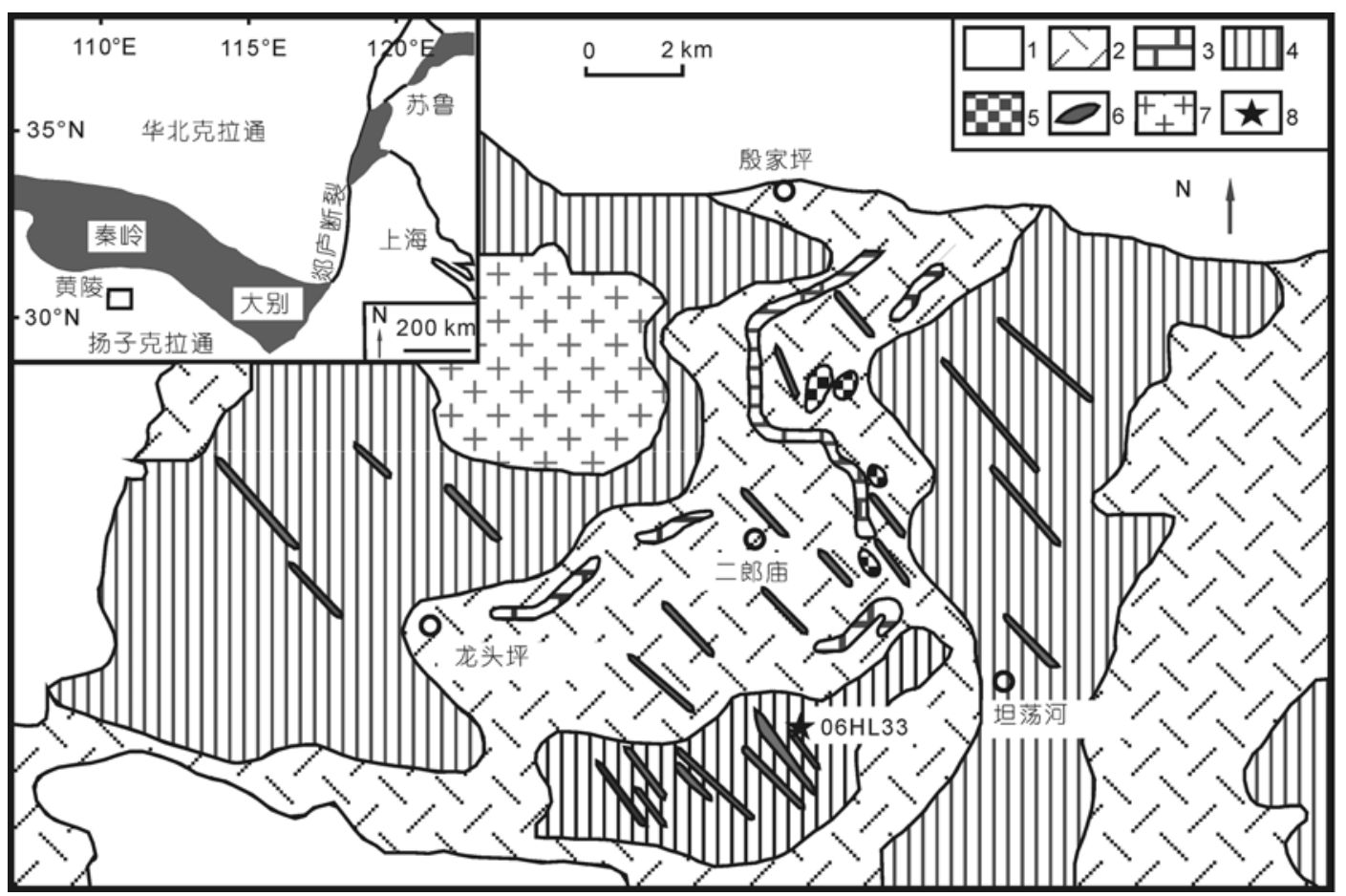

图 1 崆岭高级变质体地质简图及采样点位置

据文献[25]修改. 1，太古宙以后沉积盖层; 2 ，变沉积岩; 3, 大理岩; 4, TTG片麻岩; 5 , 超基性岩; 6, 基性岩脉;

7 , 圈椅埫钾长花岗岩; 8 , 采样点位置 
显微结构照像在西北大学大陆动力学国家重点实验 室完成, CL 分析在加载于 Quanta 400FEG 型扫描电 子显微镜上的美国 Gatan 公司的 MonoCL3+型阴极苂 光探头上完成, 分析电压和电流分别为约 $15 \mathrm{kV}$ 和 $20 \mathrm{nA}$.

锆石原位微区U-Pb同位素组成分析在中国地质 大学(武汉)地质过程与矿产资源国家重点实验室利 用LA-ICP-MS完成. 激光剥蚀系统为配备有 $193 \mathrm{~nm}$ 激光器的GeoLas 2005. 分析采用的激光剥蚀孔径为 $32 \mu \mathrm{m}$, 激光脉冲为 $10 \mathrm{~Hz}$, 能量为 $110 \mathrm{~mJ}$. ICP-MS为 美国安捷伦公司生产的Agilent7500a，实验中采用氦 气作为剥蚀物质的载气, 同位素组成用锆石 91500 作 为外标进行校正, 元素含量用NIST610 作外标、 ${ }^{29} \mathrm{Si}$ 作内标进行标定, 具体分析方法及仪器参数见文献 [28]. 锆石测定点的 $\mathrm{Pb}$ 同位素比值、 $\mathrm{U}-\mathrm{Pb}$ 表面年龄和 U-Th- $\mathrm{Pb}$ 含量采用GLITTER4.0 程序计算, 普通 $\mathrm{Pb}$ 校 正采用文献[29]方法. 锆石加权平均年龄的计算 及谐和图的绘制采用Ludwig(2001)的ISOPLOT程 序 ${ }^{[30]}$.

锆石原位 Lu-Hf 同位素测定在西北大学大陆动 力学国家重点实验室配备有 $193 \mathrm{~nm} \mathrm{ArF}$ 激光器的 Nuplasma 型 MC-ICP-MS 上完成, 分析采用的激光束 斑直径为 $44 \mu \mathrm{m}$, 剥蚀频率为 $10 \mathrm{~Hz}$, 具体分析方法
及仪器参数见文献 $[31]$. 用 ${ }^{176} \mathrm{Lu} /{ }^{175} \mathrm{Lu}=0.02669^{[32]}$ 和 ${ }^{176} \mathrm{Yb} /{ }^{172} \mathrm{Yb}=0.5886^{[33]}$ 进行同量异位干扰校正计算测 定样品的 ${ }^{176} \mathrm{Lu} /{ }^{177} \mathrm{Hf}$ 和 ${ }^{176} \mathrm{Hf} /{ }^{177} \mathrm{Hf}$ 比值. 样品测定过 程中以锆石 91500 作为标样. $\varepsilon_{\mathrm{HF}}$ 计算采用 ${ }^{176} \mathrm{Lu}$ 衰变常 数为 $1.865 \times 10^{-11} \mathrm{a}^{-1[34]}$, 球粒陨石现今值 ${ }^{176} \mathrm{Hf} /{ }^{177} \mathrm{Hf}=$ 0.282772 和 ${ }^{176} \mathrm{Lu} /{ }^{177} \mathrm{Hf}=0.0332^{[35]}$; 亏损地幔 $\mathrm{Hf}$ 模式 年龄 $\left(T_{\mathrm{DM}}\right)$ 计算采用现今亏损地幔值 ${ }^{176} \mathrm{Hf} /{ }^{177} \mathrm{Hf}=$ 0.28325 和 ${ }^{176} \mathrm{Lu} /{ }^{177} \mathrm{Hf}=0.0384^{[36]}$; 二阶段 $\mathrm{Hf}$ 模式年龄 $\left(T_{\mathrm{DM} 2}\right)$ 计算采用上地壳平均值 ${ }^{176} \mathrm{Lu} /{ }^{177} \mathrm{Hf}=0.015^{[377]}$.

\section{3 结果}

辉绿岩脉样品 $06 \mathrm{HL} 33$ 中的锆石多为半自型短柱 状、无色透明至半透明颗粒, 长度为 $80 \sim 250 \mu \mathrm{m}$, 长 宽比为 $1.5: 1 \sim 2: 1$. 锆石 CL 图像显示绝大部分锆石 具有较宽的岩浆环带或弱分带的特点, 少数颗粒具 有明显的振荡环带, 无继承型核(图 2), 属典型的岩 浆结晶产物.

用LA-ICP-MS对该样品 13 颗锆石进行了 13 个分 析点的U-Pb年龄测定, 获得的锆石U-Th-Pb同位素比 值及年龄结果见表 1 和图 3. 由表 1 可见, 该样品中 的锆石具有较低的 $U(89 \sim 451 \mathrm{ppm}, 1 \mathrm{ppm}=1$ $\left.\mu \mathrm{g} \cdot \mathrm{g}^{-1}\right)$, Th $(23 \sim 101 \mathrm{ppm})$ 含量, $\mathrm{Th} / \mathrm{U}$ 比值为 $0.07 \sim$ 0.67 , 符合岩将型锆石的特征 ${ }^{[38]} .13$ 个分析点构成一 条不一致线, 上下交点年龄分别为 $(1856 \pm 16)$ 和(64
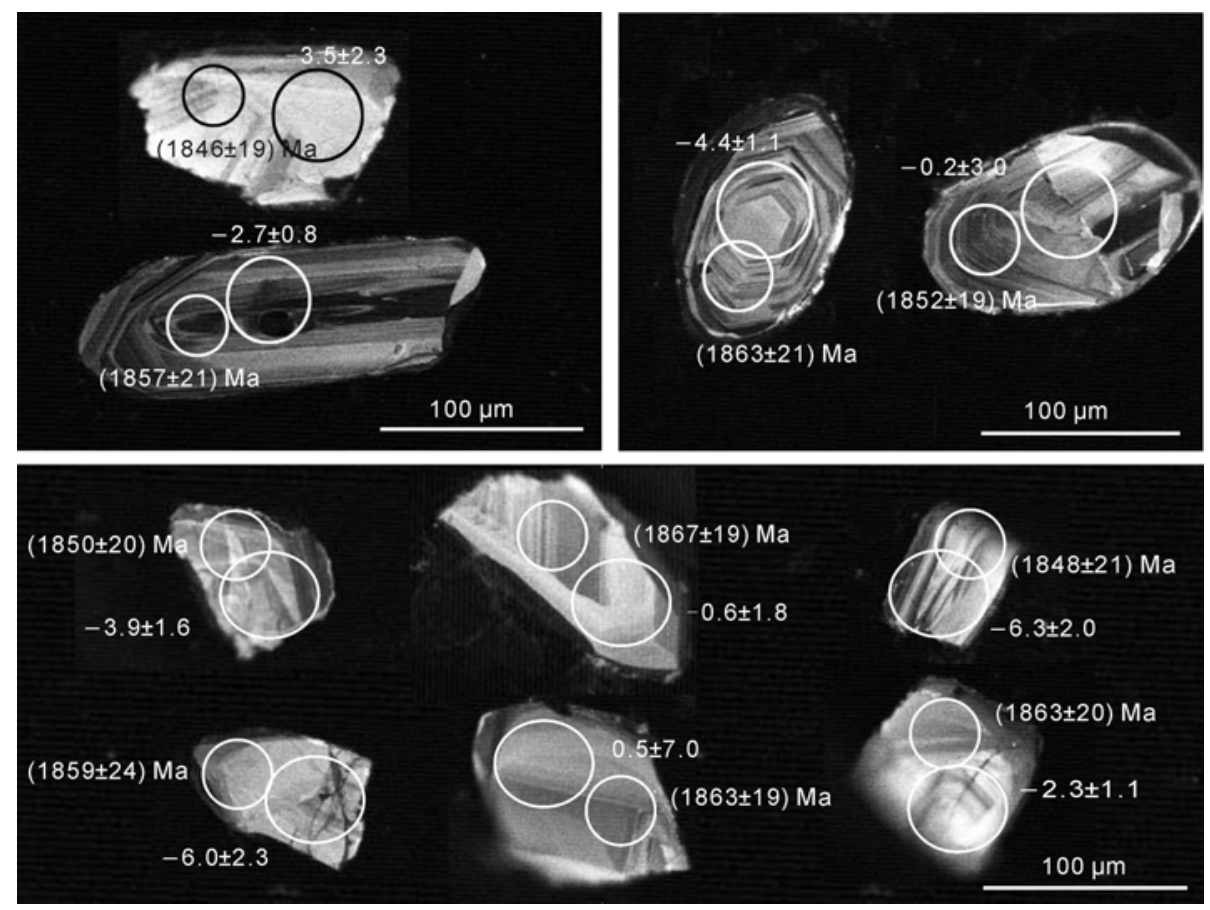

图 2 崆岭高级变质体辉绿岩中代表性锆石的 CL 图像 
表 1 辉绿岩脉样品 06HL33 中锆石 LA-ICPMS 法 U-Pb 年龄分析结果 ${ }^{\mathrm{a}}$

\begin{tabular}{|c|c|c|c|c|c|c|c|c|c|c|c|c|c|c|c|c|}
\hline \multirow{2}{*}{ 测定点 } & \multicolumn{3}{|c|}{ 元素含量/ppm } & \multirow{2}{*}{$\begin{array}{l}\mathrm{Th} / \mathrm{U} \\
\text { 比值 }\end{array}$} & \multicolumn{2}{|c|}{${ }^{207} \mathrm{~Pb} * / 206 \mathrm{~Pb}^{*}$} & \multicolumn{2}{|c|}{${ }^{207} \mathrm{~Pb} * /{ }^{235} \mathrm{U}$} & \multicolumn{2}{|c|}{${ }^{206} \mathrm{~Pb} * /{ }^{238} \mathrm{U}$} & \multicolumn{2}{|c|}{${ }^{207} \mathrm{~Pb} /{ }^{206} \mathrm{~Pb}$} & \multicolumn{2}{|c|}{${ }^{207} \mathrm{~Pb} /{ }^{235} \mathrm{U}$} & \multicolumn{2}{|c|}{${ }^{206} \mathrm{~Pb} /{ }^{238} \mathrm{U}$} \\
\hline & $\mathrm{Pb}$ & Th & $\mathrm{U}$ & & 比值 & $1 \sigma$ & 比值 & $1 \sigma$ & 比值 & $1 \sigma$ & 年龄/Ma & $1 \sigma$ & 年龄/Ma & $1 \sigma$ & 年龄/Ma & $1 \sigma$ \\
\hline 01 & 108.61 & 92 & 244 & 0.38 & 0.11417 & 0.00121 & 5.24425 & 0.06076 & 0.33307 & 0.00276 & 1867 & 19 & 1860 & 10 & 1853 & 13 \\
\hline 02 & 42.24 & 56 & 95 & 0.58 & 0.11393 & 0.00130 & 5.16637 & 0.08358 & 0.32600 & 0.00275 & 1863 & 21 & 1847 & 14 & 1819 & 13 \\
\hline 03 & 54.95 & 71 & 128 & 0.56 & 1153 & 0.00130 & 4.45070 & 0.07115 & 0.28942 & 0.00244 & 1825 & 21 & 1722 & 13 & 1639 & 12 \\
\hline 04 & 154.48 & 101 & 384 & 0.26 & 0.11321 & 0.00122 & 4.99418 & 0.06000 & 0.31934 & 0.00265 & 1852 & 19 & 1818 & 10 & 1787 & 13 \\
\hline 05 & (70.TJ & 56 & 93 & 0.01 & 0.11299 & 0.00130 & 4.93976 & 0.07597 & 0.31339 & 0.00264 & 010 & 21 & 100 & 13 & 1757 & 13 \\
\hline 06 & 175.80 & 33 & 451 & 0.07 & 0.11286 & 0.00121 & 5.16006 & 0.06051 & 0.33019 & 0.00274 & 1070 & 19 & 1070 & 10 & 1839 & 13 \\
\hline 07 & 42.49 & 53 & 102 & 0.52 & 0.11353 & 0.00131 & 4.71534 & 0.07230 & 0.30163 & 0.00255 & 1857 & 21 & 1770 & 13 & 1699 & 13 \\
\hline 08 & 105.94 & 23 & 261 & 0.09 & 0.11238 & 0.00120 & 5.16417 & 0.05749 & 0.33357 & 0.00276 & 1838 & 19 & 1847 & 9 & 1856 & 13 \\
\hline 09 & 54.18 & 71 & 132 & 0.54 & 0.11390 & 0.00123 & 4.54161 & 0.05576 & 0.28763 & 0.00239 & 1863 & 19 & 1739 & 10 & 1630 & 12 \\
\hline 10 & 56.33 & 73 & 131 & 0.56 & 0.11310 & 0.00124 & 4.49826 & 0.05978 & 0.29781 & 0.00248 & 1850 & 20 & 1767 & 11 & 1680 & 12 \\
\hline 11 & 63.71 & 91 & 151 & 0.60 & 0.11395 & 0.00124 & 4.57054 & 0.05812 & 0.28948 & 0.00241 & 1863 & 20 & 1744 & 10 & 1639 & 12 \\
\hline 12 & 12.86 & 94 & 141 & 0.67 & 0.10620 & 0.00227 & 0.95395 & 0.02557 & 0.06502 & 0.00064 & 1735 & 33 & 680 & 13 & 406 & 4 \\
\hline 13 & 39.16 & 49 & 89 & 0.55 & 0.11365 & 0.00151 & 5.16002 & 0.12412 & 0.32916 & 0.00290 & 1859 & 24 & 1846 & 20 & 1834 & 14 \\
\hline
\end{tabular}

a) $\mathrm{Pb}^{*}$ ，表示放射成因 $\mathrm{Pb}$

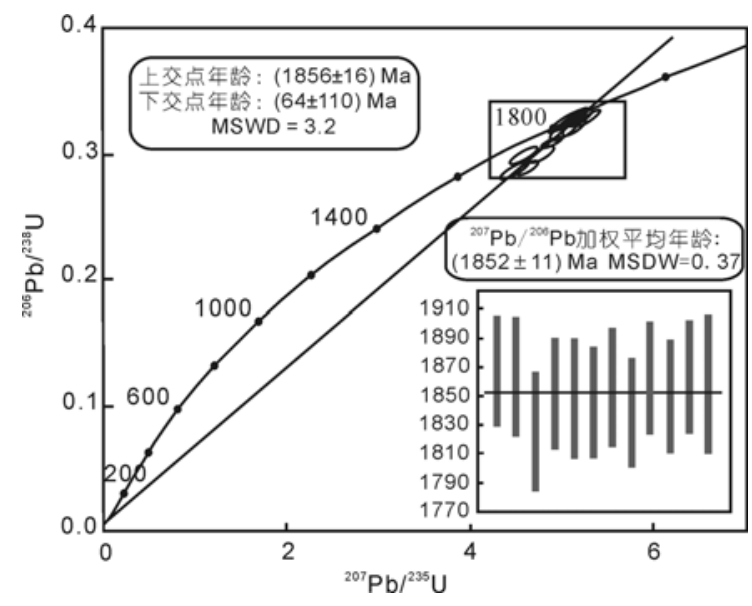

图 3 崆岭高级变质体辉绿岩锆石 U-Pb 年龄谐和图
110) Ma (MSDW = 3.2). 其中大部分点位于谐和曲线 上或其附近, 除去可能发生 $\mathrm{Pb}$ 丢失的 12 号分析点, 剩余 12 个分析点的 ${ }^{207} \mathrm{~Pb} /{ }^{206} \mathrm{~Pb}$ 加权平均年龄为 $(1852 \pm$ $11) \mathrm{Ma}(\mathrm{MSDW}=0.37)$. 该年龄与上交点年龄在误差 范围内一致, 且误差较小, 应该代表了该基性岩脉的 侵位年龄.

在 U-Pb 定年的基础上, 本文利用 LA-MC-ICPMS 对其中 10 颗锆石进行了 10 个点的 Lu-Hf 同位素 分析, 结果列于表 2 . 以 $t=1850 \mathrm{Ma}$ 计算出锆石 ${ }^{176} \mathrm{Hf} /$ ${ }^{177} \mathrm{Hf}$ 初始值为 $0.28144 \sim 0.28166$, 对应的 $\varepsilon_{\mathrm{Hf}}(t)$ 为 $-6.3 \sim 0.5$, 加权平均值为 $(-3.06 \pm 0.88)(\mathrm{MSWD}=1.01)$ (图 4(a))进行二阶段 Hf 模式年龄计算, 得到的 $T_{\mathrm{DM} 2}$ 为 $2467 \sim 2884 \mathrm{Ma}$ (平均值为 $(2693 \pm 55) \mathrm{Ma}$ )(图 4(b)).

表 2 崆岭高级变质体辉绿岩脉样品 06HL33 锆石 Lu-Hf 同位素组成

\begin{tabular}{cccccccccccc}
\hline 测定点 & ${ }^{176} \mathrm{Hf} /{ }^{177} \mathrm{Hf}$ & $2 \sigma$ & ${ }^{176} \mathrm{Lu} /{ }^{177} \mathrm{Hf}$ & ${ }^{176} \mathrm{Yb} /{ }^{177} \mathrm{Hf}$ & $\varepsilon_{\mathrm{Hf}}(0)$ & $\varepsilon_{\mathrm{Hf}}(t)$ & $2 \sigma$ & $T_{\mathrm{DM} 1} / \mathrm{Ma}$ & $2 \sigma$ & $T_{\mathrm{DM} 2} / \mathrm{Ma}$ & $2 \sigma$ \\
\hline 1 & 0.28151 & 0.000032 & 0.000800 & 0.031756 & -44.6 & -4.4 & 1.1 & 2423 & 87 & 2767 & 140 \\
2 & 0.28153 & 0.000066 & 0.000750 & 0.029291 & -43.8 & -3.5 & 2.3 & 2390 & 180 & 2716 & 288 \\
3 & 0.28162 & 0.000084 & 0.000418 & 0.017989 & -40.9 & -0.2 & 3.0 & 2259 & 227 & 2511 & 367 \\
4 & 0.28153 & 0.000022 & 0.000045 & 0.002034 & -43.9 & -2.7 & 0.8 & 2350 & 59 & 2666 & 96 \\
5 & 0.28152 & 0.000046 & 0.000528 & 0.017739 & -44.4 & -3.9 & 1.6 & 2401 & 125 & 2738 & 201 \\
6 & 0.28156 & 0.000030 & 0.000429 & 0.016439 & -43.0 & -2.3 & 1.1 & 2341 & 81 & 2643 & 131 \\
7 & 0.28144 & 0.000066 & 0.000055 & 0.002780 & -47.1 & -6.0 & 2.3 & 2472 & 176 & 2865 & 288 \\
8 & 0.28146 & 0.000056 & 0.000960 & 0.037798 & -46.3 & -6.3 & 2.0 & 2499 & 153 & 2884 & 244 \\
9 & 0.28166 & 0.000196 & 0.001248 & 0.057469 & -39.2 & 0.5 & 7.0 & 2241 & 543 & 2467 & 858 \\
10 & 0.28164 & 0.000052 & 0.001338 & 0.056253 & -40.1 & -0.6 & 1.8 & 2283 & 144 & 2533 & 227 \\
\hline
\end{tabular}

a) $\mathrm{Hf}$ 初始比值采用 $t=1850 \mathrm{Ma}$ 进行计算 

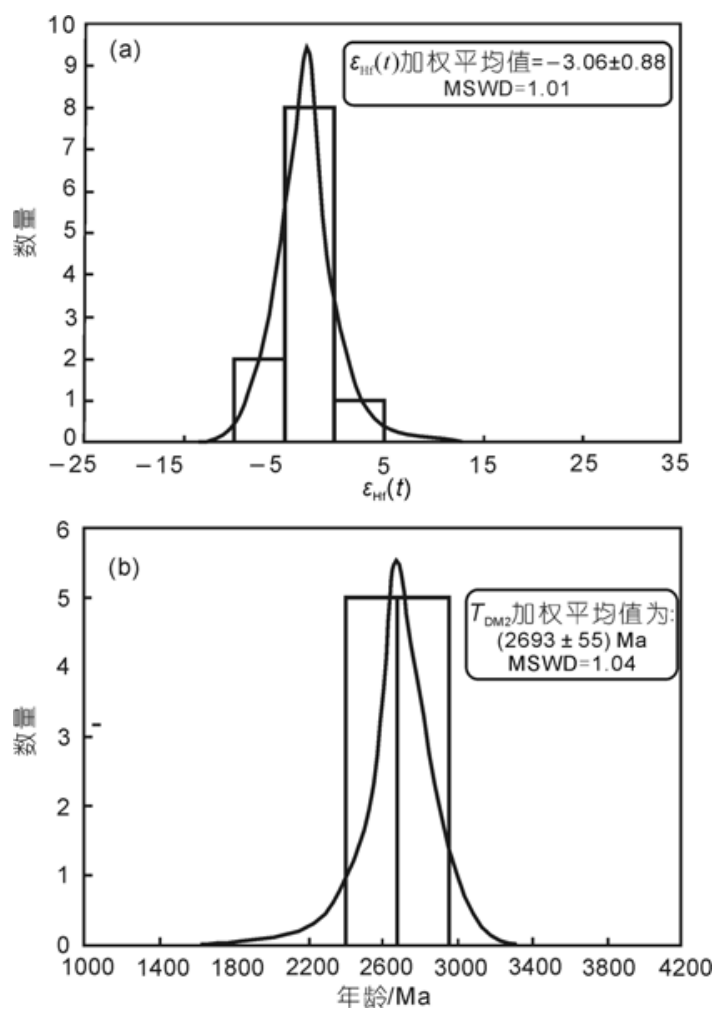

图 4 崆岭高级变质体辉绿岩锆石 $\varepsilon_{\mathrm{Hf}}(t)$ 值 $(\mathrm{a})$ 和二阶段 Hf 模式年龄(b)柱状分布图

\section{4 讨论}

由于基性岩脉中的锆石既可能是从围岩中捕获 而来, 也可以是从基性岩浆中结晶的, 而只有从基性 岩浆中结晶的锆石的年龄才能代表基性岩脉侵入的 准确年龄，因此，对于基性岩脉年龄的测定必须选择 原生的岩浆锆石进行测定 ${ }^{[39]}$. 本文样品的锆石具有 较宽的岩浆环带或无明显分带特征，应为基性岩浆 中结晶的锆石 ${ }^{[38]}$; 部分颗粒具有清楚的振荡环带, 这种类型的锆石在基性岩脉中也有报道 ${ }^{[40 \sim 42]}$. 绝大 部分锆石具有较低的 $T h, U$ 含量和较高的 $T h / U$ 比值 $(>0.2)$, 这些特征与基性岩浆中结晶的锆石特征一致; 两个锆石颗粒的 $\mathrm{Th} / \mathrm{U}$ 比值小于 0.1 , 这样的结果在前 人对基性岩脉的研究中同样也有报道 ${ }^{[3,43]}$. 同时该基 性岩脉的直接围岩为约 $2.9 \mathrm{Ga}$ 的TTG片麻岩 ${ }^{[23,25]}$, 而 附近的变沉积岩的碎屑锆石和变质锆石 $U-\mathrm{Pb}$ 年龄也 都大于 $1.95 \mathrm{Ga}^{[24]}$, 因此本文分析的基性岩脉样品中 的锆石捕获围岩约 $1.85 \mathrm{Ga}$ 捕掳晶锆石的可能性较小. 本文基性岩脉中锆石的 ${ }^{176} \mathrm{Hf} /{ }^{177} \mathrm{Hf}$ 初始值为 $0.28144 \sim 0.28166$, 对应的 $\varepsilon_{\mathrm{Hf}}(t)$ 为 $-6.3 \sim 0.5$, 加权平
均值为 $(-3.06 \pm 0.88)(M S W D=1.01)$ (图 4(a)), 显示 其岩浆源区可能为相对富集的岩石圈地幔. 本文进 行年龄测定的锆石为基性岩脉中结晶的锆石颗粒. 这些岩浆锆石的 ${ }^{207} \mathrm{~Pb} /{ }^{206} \mathrm{~Pb}$ 加权平均年龄为 $(1852 \pm$ 11) $\mathrm{Ma}(\mathrm{MSDW}=0.37)$, 与上交点年龄一致且误差较 小, 因此, $(1852 \pm 11) \mathrm{Ma}$ 应该代表了基性岩脉侵位 的准确年龄.

Columbia超大陆的存在已经得到较为广泛的认 可, 时代约为 $2.1 \sim 1.8 \mathrm{Ga}$ 的全球性的碰撞造山事件 代表了其聚合的主要过程 ${ }^{[44]}$. 最新研究表明, 扬子板 块不同地区存在 $1.8 \sim 2.0 \mathrm{Ga}$ 构造热事件的年代学记 录. Zhang等人 ${ }^{[23,24]}$ 从崆岭高级变质体的变质岩和混 合岩中获得了一批 $1.95 \sim 2.0 \mathrm{Ga}$ 的锆石 $\mathrm{U}-\mathrm{Pb}$ 年龄; Qiu等人 ${ }^{[45]}$ 从奥长花岗岩和负片麻岩的变质锆石中获 得了(1992 \pm 16$)$ 和(1958 \pm 15$)$ Ma的谐和年龄; 凌文 黎等人 ${ }^{[46]}$ 从斜长角闪岩和副片麻岩中获得 $\mathrm{Sm}-\mathrm{Nd}$ 矿 物等时线年龄为 $(1958 \pm 15)$ 和 $(1939 \pm 44) \mathrm{Ma}$. 同时 扬子板块其他地方存在相似的年龄记录 ${ }^{[19,20]}$, 这些 年代学记录揭示出扬子板块存在这一时代为约 2.0 $\mathrm{Ga}$ 的碰撞造山作用, 并且可能与Columbia超大陆的 聚合有关 ${ }^{[19,23,24]}$.

基性岩脉是伸展作用背景下岩石圈地幔部分熔 融作用的产物, 因此是区域伸展作用的重要标志 ${ }^{[2]}$. 基性岩脉既可以形成于与俯冲作用有关的弧后伸展 作用 ${ }^{[12,47]}$, 也可以形成于板内伸展的构造背景 ${ }^{[2,11]}$. 扬子板块与Columbia超大陆聚合作用有关的造山事 件发生在约 $2.0 \mathrm{Ga}$, 而本文报道的基性岩脉的形成时 间约为 $1.85 \mathrm{Ga}$, 明显晚于碰撞造山作用事件发生的 时间, 它们不可能是同一构造作用过程的产物. 此外, 崆岭地区出露的圈椅埫岩体是年龄约为 $1.85 \mathrm{Ga}$ 的 $\mathrm{A}$ 型花岗岩 ${ }^{[48]}$, 它是碰撞后板内伸展环境中岩浆作用 的产物. 这些 $\mathrm{A}$ 型花岗岩具有非常负的 $\varepsilon_{\mathrm{Hf}}(t)$ 值 $-27.0 \sim-16.7$, 指示其岩浆源区比基性岩墙的要富集 得多, 可能是古老太古宙基底 ${ }^{[49]}$. 不过, 这些基性和 酸性侵入体都形成于碰撞后板内伸展的构造背景, 指示在约 $1.85 \mathrm{Ga}$ 扬子板块发生了由碰撞挤压向伸展 作用的构造转换.

基性岩墙中锆石的产出指示其结晶岩浆处于 $\mathrm{Zr}$ 饱和状态，因此这种镁铁质岩浆可能由相对难熔的 弧下岩石圈地幔在弧陆碰撞之后发生熔融所形成的 [50]. 形成于板内的基性岩脉与大陆地壳或岩石圈伸 展作用产生的刚性破裂相联系 ${ }^{[1]}$ ，它们是大陆板块刚 


\title{
性化及其古应力状态最直接的地质标志 ${ }^{[7,51]}$. 本文研 以致能产生大规模的脆性破裂，并可能发生地幔对 究结果显示, 扬子板块发育时代为 $1.85 \mathrm{Ga}$ 的基性岩 流作用，因此我们推测扬子板块克拉通化的完成应 脉，表明该时期扬子板块已经具备足够的刚性特征, 不晚于 $1.85 \mathrm{Ga}$.
}

\begin{abstract}
评审人提出了建设性的修改意见, LA-ICPMS 锆石 U-Pb 年龄测定得到陈海红老师的帮助, 锆石 CL 照像得到西 北大学大陆动力学国家重点实验室弓虎军博士的帮助, 锆石 Lu-Hf 同位素测试得到宗春蕾老师的指导和帮助, 在此一并表示衷心的感谢.
\end{abstract}

\section{参考文献}

1 Mathieu L, van Wyk de Vries B, Holohan E P, et al. Dykes, cups, saucers and sills: Analogue experiments on magma intrusion into brittle rocks. Earth Planet Sci Lett, 2008, 271: 1-13 $\underline{\text { [doi] }}$

2 Williams H, Turner S, Kelley S, et al. Age and composition of dikes in Southern Tibet: New constraints on the timing of east-west extension and its relationship to postcollisional volcanism. Geology, 2001, 29: 339-342[10i]

3 Kullerud K, Skjerlie K P, Corfu F, et al. The 2.40 Ga Ringvassøy mafic dikes, West Troms Basement Complex, Norway: The concluding act of early Palaeoproterozoic continental breakup. Precambrian Res, 2006, 150: 183-200[doi]

4 Ernst R E, Buchan K L, West T D, et al. Diabase (Dolerite) dike swarms of the world. Open File-Geol Surv Can, 1996, 3241: 1-104

5 Yale L B, Carpenter S J. Large igneous provinces and giant dike swarms: Proxies for supercontinent cyclicity and mantle convection. Earth Planet Sci Lett, 1998, 163: 109-122[doi]

6 Condie K C. Episodic continental growth and supercontinents: A mantle avalanche connection? Earth Planet Sci Lett, 1998, 163: 9710810.1016/S0012-821X(98)00178-2

7 李江海, 何文渊, 钱祥麟. 元古代基性岩墙群的成因机制、构造背景及其古板块再造意义. 高校地质学报, 1997, 3(3): 272一-281

8 Zheng Y F, Fu B, Gong B, et al. Stable isotope geochemistry of ultrahigh pressure metamorphic rocks from the Dabie-Sulu orogen in China: Implications for geodynamics and fluid regime. Earth Sci Rev, 2003, 62: 105-161 [doi]

9 Wu Y B, Zheng Y F, Zhao Z F, et al. U-Pb, Hf and O isotope evidence for episodes of fluid-assisted zircon growth in marble-hosted eclogites from the Dabie orogen. Geochim Cosmochim Acta, 2006, 70: 3743 - 3761 [doi]

10 Li S G, Jagoutz E, Lo C H, et al. Sm/Nd, Rb/Sr, and ${ }^{40} \mathrm{Ar} /{ }^{39} \mathrm{Ar}$ isotopic systematics of the ultrahigh-pressure metamorphic rocks in the Dabie-Sulu belt Central China: A retrospective view. Int Geol Rev, 1999, 41: 1114-1124

11 Wang Y J, Fan W M, Zhang Y H. Geochemical, ${ }^{40} \mathrm{Ar} /{ }^{39} \mathrm{Ar}$ geochronological and Sr-Nd isotopic constraints on the origin of Paleoproterozoic mafic dikes from the southern Taihang Mountains and implications for the ca. $1800 \mathrm{Ma}$ event of the North China Craton. Precambrain Res, 2004, 135: 55 - 79 [doi]

12 Wang Y J, Zhao G C, Fan W M, et al. LA-ICP-MS U-Pb zircon geochronology and geochemistry of Paleoproterozoic mafic dikes from western Shandong Province: Implications for back-arc basin magmatism in the Eastern Block, North China Craton. Precambrain Res, 2007, 154: 107124 [doi]

13 Zhai M G, Liu W J. Palaeoproterozoic tectonic history of the North China craton: A review. Precambrain Res, 2003, 122: 183-199[doi]

14 李江海, 侯贵廷, 黄雄南, 等. 华北克拉通对前寒武纪超大陆旋回的基本制约. 岩石学报, 2001, 17(2): 177一186

15 Guo J H, Sun M, Chen F K, et al. Sm-Nd and SHRIMP U-Pb zircon geochronology of high-pressure granulites in the Sanggan area, North China Craton: Timing of Paleoproterozoic continental collision. J Asian Earth Sci, 2005, 25: 629-642[doi]

16 Wan Y S, Song B, Liu D Y, et al. SHRIMP U-Pb zircon geochronology of Palaeoproterozoic metasedimentary rocks in the North China Craton: Evidence for a major Late Palaeoproterozoic tectonothermal event. Precambrain Res, 2006, 149: 249—271 [doi]

17 Peng P, Zhai M G, Ernst R E, et al. 1.78 Ga large igneous province in the North China craton: The Xiong'er Volcanic Province and the North China dyke swarm. Lithos, 2008, 101: 260-280[doi]

18 Zhao G C, Sun M, Wilde S A, et al. Late Archean to Paleoproterozoic evolution of the North China Craton: Key issues revisited. Precambrain Res, 2005, 136: 177 -206[doi]

19 Wu Y B, Zheng Y F, Liu Y S, et al. Zircon U-Pb age and trace element evidence for Paleoproterozoic granulite facies metamorphism and Archean crustal rocks in the Dabie Orogen. Lithos, 2008, 101: 308 - 322[doi]

20 Zheng J P, Griffin W L, O’Reilly S Y, et al. Widespread Archean basement beneath theYangtze craton. Geology, 2006, 34: 417-420[doi]

21 沈其韩，耿元生，宋彪，等. 华北和扬子陆块及秦岭-大别造山带地表和深部太古宙基底的新信息. 地质学报, 2005, 79(5): 616-627

22 郑永飞. 新元古代岩浆活动与全球变化. 科学通报, 2003, 48(16): 1705-1720 
23 Zhang S B, Zheng Y F, Wu Y B, et al. Zircon isotope evidence for $\geqslant 3.5$ Ga continental crust in the Yangtze craton of China. Precambrian Res, 2006, 146: 16-34[doi]

24 Zhang S B, Zheng Y F, Wu Y B, et al. Zircon U-Pb age and Hf-O isotope evidence for Paleoproterozoic metamorphic event in South China. Precambrain Res, 2006, 151: 265-288[doi]

25 Gao S, Ling W L, Qiu Y M, et al. Contrasting geochemical and Sm-Nd isotopic compositions of Archean metasediments from the Kongling high-grade terrane of the Yangtze craton: Evidence for cratonic evolution and redistribution of REE during crustal anatomies. Geochim Cosmochim Acta, 1999, 63: 2071-2088[doi]

26 袁海华, 张志兰, 刘炜, 等. 直接蒸发单颗粒锆石测定 ${ }^{207} \mathrm{~Pb} /{ }^{206} \mathrm{~Pb}$ 年龄的方法. 矿物岩石, 1991, 11(2): 72-79

27 Li X H, Li Z X, Ge W C, et al. Neoproterozoic granitoids in South China: Crustal melting above a mantle plume at ca. 825 Ma? Precambrian Res, 2003, 122: 45-83[doi]

28 袁洪林, 吴福元, 高山，等. 东北地区新生代侵入体的锆石激光探针 U-Pb 年龄测定与稀土元素成分分析. 科学通报, 2003, 48(14): 1511 -1520

29 Andersen T. Correction of common lead in U-Pb analyses that do not report ${ }^{204} \mathrm{~Pb}$. Chem Geol, 2002, 192: 59-79[doi]

30 Ludwig K R. Users manual for Isoplot/Ex (rev.2.49): A Geochronological Toolkit for Microsoft Excel. Berkeley Geochron Cent Spec Publ, 2001. $1-55$

31 Yuan H L, Gao S, Dai M N, et al. Simultaneous determinations of U-Pb age, Hf isotopes and trace element compositions of zircon by excimer laser ablation quadrupole and multiple collector ICP-MS. Chem Geol, 2008, 247: 100 - 118[doi]

32 De Bievre P, Taylor P D P. Table of the isotopic composition of the elements. Int J Mass Spectrom Ion Process, 1993, 123: 1-149[doi]

33 Chu M F, Chung S L, Song B, et al. Zircon U-Pb and Hf isotope constraints on the Mesozoic tectonics and crustal evolution of southern Tibet. Geology, 2006, 34: 745-748[doi]

34 Scherer E, Munker C, Mezger K. Calibration of the lutetium-hafnium clock. Science, 2001, 293: 683-687[doi]

35 Blichert-Toft J, Albarede F. The Lu-Hf geochemistry of chondrites and the evolution of the mantle-crust system. Earth Planet Sci Lett, 1997, 148: $243-258$ [doi]

36 Vervoort J D, Blichert-Toft J. Evolution of the depleted mantle: Hf isotope evidence from juvenile rocks through time. Geochim Cosmochim Acta, 1999, 63: 533-556[doi]

37 Griffin W L, Wang X, Jackson S E, et al. Zircon chemistry and magma mixing, SE China: in-situ analysis of Hf isotopes, Tonglu and Pingtan igneous complexes. Lithos, 2002, 61: 237-269[doi]

38 吴元保, 郑永飞. 锆石成因矿物学研究及其对 U-Pb 年龄解释的制约. 科学通报, 2004, 49(16): 1859-1604

39 侯贵廷, 刘玉琳, 李江海, 等. 关于基性岩墙群的 U-Pb SHRIMP 地质年代学的探讨一以鲁西莱芜辉绿岩岩墙为例. 岩石矿物学杂 志, 2005, 3(24): 179-185

40 Liu S, Hu R Z, Gao S, et al. Zircon U-Pb geochronology and major, trace elemental and Sr-Nd-Pb isotopic geochemistry of mafic dykes in western Shandong Province, East China: Constrains on their petrogenesis and geodynamic significance. Chem Geol, 2008, 255: 325-349

41 林广春, 李献华, 李武显. 川西新元古代基性岩墙群的 SHRIMP 锆石 U-Pb 年龄、元素和 Nd-Hf 同位素地球化学: 岩石成因与构造 意义. 中国科学 D 辑: 地球科学, 2006,36(7): 630-645

42 Kröner A, Wilde S A, Zhao G C, et al. Zircon geochronology and metamorphic evolution of mafic dykes in the Hengshan Complex of northern China: Evidence for late Palaeoproterozoic extension and subsequent high-pressure metamorphism in the North China Craton. Precambrain Res, 2006, 146: 45-67[doi]

43 Hou G T, Liu Y L, Li J H. Evidence for $\sim 1.8$ Ga extension of the Eastern Block of the North China Craton from SHRIMP U-Pb dating of mafic dyke swarms in Shandong Province. J Asian Earth Sci, 2006, 27: 392 - 401 [doi]

44 Zhao G C, Sun M, Wilde S A, et al. A Paleo-Mesoproterozoic supercontinent: Assembly, growth and breakup. Earth Sci Rev, 2004, 67: 91-123

45 Qiu Y M, Gao S, McNaughton N J, et al. First evidence of $>3.2$ Ga continental Crust in the Yangtze craton of South China and its implications for Archean Crustal evolution and phanerozoic tectonics. Geology, 2000, 28(1): 11-14 [doi]

46 凌文黎, 高山, 张本仁, 等. 扬子陆核古元古代晚期构造热事件与扬子克拉通演化. 科学通报, 2000, 45(21): 2343-2348

47 Cadman A C, Tarney J, Bridgwater D, et al. The petrogenesis of the Kangâmiut dike swarm, W. Greenland. Precambrain Res, 2001, 105: 183203 [doi]

48 熊庆, 郑建平, 余淳梅, 等. 宜昌圈椅埫 A 型花岗岩锆石 U-Pb 年龄和 Hf 同位素与扬子大陆古元古代克拉通化作用. 科学通报, 2008 , 53(22): 2782-2792

49 Zhang S B, Zheng Y F, Zhao Z F, et al. Neoproterozoic anatexis of Archean lithosphere: Geochemical evidence from felsic to mafic intrusives at Xiaofeng in the Yangtze George, South China. Precambrain Res, 2008, 163: 210-238 [doi]

50 Zheng Y F, Wu R X, Wu Y B, et al. Rift melting of juvenile arc-derived crust: Geochemical evidence from Neoproterozoic volcanic and granitic rocks in the Jiangnan Orogen, South China. Precambrain Res, 2008, 163: 351-383 [doi]

51 Desmond E M, John R B, John W, et al. Creation of a continent recorded in zircon zoning. Geology, 2008, 36(3): 239—242[doi] 